\title{
Characteristics of Corneal Subbasal Nerves in Different Age Groups: An in vivo Confocal Microscopic Analysis
}

\author{
Chareenun Chirapapaisan $\mathbb{D}^{1}$ \\ Sittiphong Thongsuwan' \\ Niphon Chirapapaisan' \\ Pratuangsri Chonpimai' \\ Anupong Veeraburinon ${ }^{2}$ \\ 'Department of Ophthalmology, Faculty \\ of Medicine Siriraj Hospital, Mahidol \\ University, Bangkok, Thailand; ${ }^{2}$ Research \\ Division, Faculty of Medicine Siriraj \\ Hospital, Mahidol University, Bangkok, \\ Thailanda
}

Purpose: To determine the normative characteristics of corneal subbasal nerves in different age groups using laser scanning in vivo confocal microscopy (IVCM).

Patients and Methods: This descriptive observational study recruited healthy subjects (aged 20-60 years) from Siriraj Health-Screening Center. Excluded were individuals who had abnormal ocular symptoms, previous ocular surgery, a history of any diseases related to systemic and/or corneal neuropathy, or abnormal corneal sensitivity. Corneal IVCM (HRT3/ Rostock Corneal Module) was performed at the central cornea to analyze the subbasal nerve plexus. The corneal nerve characteristics, comprising the number and density of nerves (main nerve trunks, branches, and total nerves) were analyzed using the NeuronJ program, and the corneal nerve tortuosity was graded. The correlations between the subbasal nerve density, tortuosity and age were then analyzed.

Results: Eighty subjects were enrolled, with twenty in each of four age groups $(20-30,>30$ $40,>40-50$, and $>50-60$ years). Overall, the mean number and density of main nerve trunks were $27.93 \pm 0.81 / \mathrm{mm}^{2}$ and $11.22 \pm 0.30 \mathrm{~mm} / \mathrm{mm}^{2}$, respectively. As of the nerve branches, the average number and density were $103.56 \pm 2.37 / \mathrm{mm}^{2}$ and $9.15 \pm 0.30 \mathrm{~mm} / \mathrm{mm}^{2}$, respectively. The total nerve density was $20.37 \pm 0.39 \mathrm{~mm} / \mathrm{mm}^{2}$. There were no significant differences between subbasal nerve parameters of the four age groups. It is noteworthy that $65 \%$ of the subjects aged over 40 years revealed high-grade nerve tortuosity.

Conclusion: The corneal subbasal nerve numbers and densities were not significantly different among a healthy population aged 20-60 years. However, there was a trend towards high tortuosity of the corneal nerve in people aged over 40 years.

Keywords: in vivo confocal microscopy, IVCM, corneal subbasal nerves, normal characteristics, age

\section{Introduction}

The cornea is considered the most innervated structure in the human body. ${ }^{1}$ Corneal innervation, derived from the trigeminal nerve, plays an important role in the maintenance of the anatomy and physiology of the cornea. An intact corneal innervation results in a normal corneal sensitivity and blink reflex, which is essential for the protective mechanism of the eye. ${ }^{1}$ Moreover, all trophic functions of the cornea and ocular surface, which include tear production, epithelial proliferation, corneal wound healing and the remodeling process rely on the corneal nerve supply. ${ }^{2}$ Neurotrophic Keratopathy (NK), a well-known condition, is a paradigm of corneal disease caused by an impairment of the trigeminal nerve that leads
Correspondence: Niphon Chirapapaisan Department of Ophthalmology, Faculty of Medicine Siriraj Hospital, Mahidol

University, Bangkok, Thailand

Tel +662 4198037

Fax +662414 I232

Email niphon.chi@mahidol.ac.th 
to corneal denervation. ${ }^{3}$ Debilitating corneal innervation contributes to a decrease in corneal sensitivity and an abnormal corneal repair process. In such a case, the ocular surface integrity gradually degenerates along with a deterioration in the restorative process. Ultimately, a persistent corneal epithelial defect, progressive stromal thinning, and corneal perforation may arise. Although the prevalence of NK has not yet explicitly determined, ${ }^{4}$ prolonged or severe NK is a challenging and almost irretrievable condition, posing a risk of corneal blindness. It is evidenced that the two most common causes of NK are herpetic keratitis and post-surgical damage of trigeminal nerve. ${ }^{4}$ Other etiologies of NK can be attributed to a direct corneal nerve injury (such as a trauma or burn), a severe corneal infection, keratorefractive surgery, and major corneal surgery including transplantation. ${ }^{3}$ Moreover, chronic-repetitive corneal inflammation arising from contact lens use, recurrent corneal erosion, and dry eye $\mathrm{e}^{5,6}$ also impair the corneal subbasal nerves and eventually result in NK.

Recently, there have been extensive studies on NK. Several publications have reported that not merely corneal diseases, systemic diseases such as diabetes mellitus, multiple sclerosis, leprosy, vitamin A deficiency, amyloidosis, and central nervous system related diseases could induce neuropathic cornea. ${ }^{3,6,7}$ Since numerous ocular and systemic diseases can instigate corneal denervation, this has heightened the need for investigators to direct their efforts to the search for a method to identify patients at risk of $\mathrm{NK}$, in order to prevent future sequelae. To date, the standard diagnosis of NK has been based on clinical information obtained from a patient's history; general physical exam; evidence of ocular manifestations that are characteristic of $\mathrm{NK}_{;}^{2,3}$ and the confirmation of low corneal sensitivity, which is commonly tested with a CochetBonnet esthesiometer (Luneau Ophtalmologie, Chartres, France). ${ }^{8,9}$ In fact, the severity of NK varies with the degree of subbasal nerve damage. Since the diminishment of the nerves in the early stage of NK is minimal, clinical changes are not detectable, and the corneal sensitivity remains preserved. As the disease progressively worsens, leading to a pronounced nerve decline, the clinical presentations of NK become distinct and a diagnosis can be established. This was affirmed in a study by Hamrah et al.${ }^{10}$ They demonstrated that a decrease in corneal sensation could be clinically detected by a Cochet-Bonnet esthesiometer when the density of corneal subbasal nerves analyzed by a slit-scanning in vivo confocal microscope decreased to less than $5.22 \mathrm{~mm} / \mathrm{mm}^{2} \quad(835 \mu \mathrm{m} /$ frame $)$ whereas the normal nerve density of their healthy controls was about $14.11 \mathrm{~mm} / \mathrm{mm}^{2}(2258.4 \mu \mathrm{m} /$ frame $)$. Given that the corneal nerves are invisible, a solely conventional clinical approach is unable to quantify them and hence is inadequate for the recognition of early or subclinical NK.

With many technological advances of the recent decades in the field of ophthalmology, a number of imaging devices have come forth that enable advanced investigations, particularly by means of the visualization of ocular structures at the cellular level. As a result, otherwise invisible microstructural alterations are able to be exposed, and the diagnosis and management of several ophthalmic conditions are greatly supported by imaging systems. Of those, in vivo confocal microscopy (IVCM) is crucial, facilitating investigation of the cornea and ocular surface diseases. Since corneal IVCM provides visualization of the intracorneal cellular structures layer by layer, ${ }^{6,11,12}$ a noninvasive and real-time exploration of the entire cornea is feasible. ${ }^{13}$ Extensive analyses have been made of a wide variety of information acquired from IVCM, such as corneal structural changes; subbasal nerve morphology and density; and inflammatory reactions including some pathogens of infectious keratitis. ${ }^{6,12,14-16}$ The results of those studies have assisted in the prompt diagnosis and timely management of a range of diseases.

Given the importance and advantages of early recognition of corneal nerve alterations, the current research is aimed to analyze the corneal subbasal nerves. The specific goal was to determine the normal features of the nerves in terms of their density and tortuosity in an adult-healthy population, using in vivo laser scanning confocal microscopy. Previous studies found no correlation between corneal nerve density and age. ${ }^{17,18}$ Besides, ocular nerve density had no effect on laterality. ${ }^{19,20}$ On the other hand, some researchers revealed a correlation between corneal nerve density loss and age. ${ }^{19-21}$ Therefore, we anticipated that the results of this study, which we intentionally screened from absolutely healthy subjects, limited the age range of participants and compared with those of age matched controls, can be generally applied as a standard baseline characteristic of corneal subbasal nerves to distinguish otherwise invisible abnormalities or degenerative nerve changes from normal nerve findings at each age. In turn, this may support the diagnosis and/or prognostication of some subclinical NK-related conditions and expedite the management of particular ocular and systemic diseases. 


\section{Materials and Methods}

This observational, descriptive study was conducted in accordance with the Declaration of Helsinki and initiated after receiving approval from the Ethics Committee of the Siriraj Institutional Review Board, Faculty of Medicine Siriraj Hospital, Mahidol University, Thailand. The study population was informed about the purpose of the study and recruited from the Siriraj Health-Screening Center. The subjects were aged 20-60 years and were healthy, having no symptoms or signs of systemic diseases, no diabetes, and normal general blood tests. Individuals were invited to participate in the research if they did not report any history of an eye and/or systemic condition that could affect the corneal nerves (such as symptomatic dry eye; corneal ulcer; corneal dystrophy; previous ocular injury or surgery; glaucoma; current use of ocular medication; contact lens use; stroke; and central nervous systemrelated diseases, including connective tissue diseases). The subjects gave their written permission to participate.

\section{Participants}

All subjects underwent a complete eye examination using a slit-lamp biomicroscope. Fluorescein staining and the tear film breakup time test were performed to check the ocular surface integrity and dryness. The corneal sensitivity was measured at the central cornea using a CochetBonnet esthesiometer (Luneau Ophthalmologie, Chartres, France), following a standard technique. ${ }^{10}$ Only participants confirmed as having a normal eye examination with normal corneal sensation (60 $\mathrm{mm}$ of Cochet-Bonnet esthesiometer) were sent for a corneal confocal microscopic analysis; the remainder were excluded. The included subjects were classified into four groups based on their age (20-30, > 30-40, > 40-50, and $>50-60$ years).

\section{IVCM Analysis}

Laser scanning in vivo confocal microscopy (IVCM) was performed bilaterally in all subjects using a Retina Tomograph 3 machine with a Rostock Cornea Module (HRT3/RCM; Heidelberg Engineering GmbH., Heidelberg, Germany). Only one eye of each participant was subsequently randomly selected for analysis. The confocal device provided $800 \times$ magnified images of the scanned objects in real time. Each confocal image showed a four $\mu \mathrm{m}$ axial resolution with a field of view of $400 \mu \mathrm{m}$ by $400 \mu \mathrm{m} .{ }^{13}$ Before starting the corneal scan, a disposable, sterile, polymethylmethacrylate cap (TomoCap;
Heidelberg Engineering GmbH., Heidelberg, Germany) was filled with a lubricating carbomer gel $(0.2 \%$ Vidisic Gel; Bausch and Lomb, Berlin, Germany) and mounted to the optics of the confocal microscope. The gel was also applied on the subject-contact side of the TomoCap to provide lubrication during the IVCM scan. Anesthetic eye drops $(0.5 \%$ tetracaine hydrochloride; Alcon, Texas, USA) were instilled into both eyes of the subject. A softdaily contact lens was then applied to each eye to protect the corneal surfaces, followed by an overlay of carbomer gel to provide moisture and lubrication. After positioning the subject's head on the chin rest of the device, the subject was requested to keep the eyes open and look straight ahead during the one-minute examination of each eye. An experienced technician identified the central area of the cornea from outside and double-checked it by utilizing the digital camera screen mounted on the microscope. The IVCM scanning was subsequently initiated by manually advancing the microscope optics (on which was attached the TomoCap) until the subject's eye was touched. Multiple, full-thickness, corneal scans were performed using the sequence-mode function of the confocal device. The corneal subbasal nerve plexus, located between the basal epithelial and Bowman's layer, was particularly focused. The five best-quality images of the subbasal nerves were randomly selected as representative images for later analysis. Two masked, independent observers (CC and ST) quantified the subbasal corneal nerves using the semiautomated, nerve-tracing program, NeuronJ, version 1.4.3, a plug-in for ImageJ (http://rsb.info.nih.gov/ ij/http://rsb.info.nih.gov/ij/; http://www.imagescience.org/ meijering/software/neuronj/). ${ }^{22}$ The nerve parameters (the numbers and densities of the main nerve trunks, nerve branches, and total nerves) were recorded against each subject's age group. In addition, the tortuosity of the subbasal nerves was graded into five categories $(0-4)$ in accordance with the report of Oliviera-Soto et al. ${ }^{23}$ Lastly, the nerve parameters and tortuosity of the four age groups were compared.

\section{Statistical Analysis}

Statistical analyses were performed with IBM SPSS Statistics for Windows, version 23 (IBM Corp., Armonk, N.Y., USA). The normality of the demographic data of the subjects was analyzed using the Kolmogorov-Smirnov test. The reliabilities of the nerve quantifications and tortuosity gradings of the two independent observers were evaluated using an intraclass correlation coefficient (ICC), 
Table I Demographic Data of 80 Healthy Subjects, Aged Between 20-60 Years

\begin{tabular}{|c|c|c|c|c|c|}
\hline & \multicolumn{4}{|l|}{ Age (Years) } & \multirow[t]{2}{*}{ Total } \\
\hline & $20-30$ & $>30-40$ & $>40-50$ & $>50-60$ & \\
\hline Mean age $( \pm S D)$ & $24.85 \pm 2.45$ & $35.25 \pm 2.76$ & $44.05 \pm 2.78$ & $54.90 \pm 2.77$ & \\
\hline Number & 20 & 20 & 20 & 20 & 80 \\
\hline Male (mean age) & $10(24.3)$ & $10(34.5)$ & $10(43.5)$ & $10(54.5)$ & 40 \\
\hline Female (mean age) & $10(25.4)$ & $10(36.0)$ & $10(44.6)$ & $10(55.3)$ & 40 \\
\hline \multicolumn{6}{|l|}{ Laterality } \\
\hline Right eye & 10 & 10 & 10 & 10 & 40 \\
\hline Left eye & 10 & 10 & 10 & 10 & 40 \\
\hline
\end{tabular}

Abbreviation: SD, Standard deviation.

for which an excellent correlation is deemed to be represented by an ICC $>0.75 .^{24}$ Only the reliable nerve data from the two observers were averaged and recorded as mean and standard error of mean (SEM); the uneven data were reevaluated until congruent outcomes were achieved. The correlations between the subbasal nerve densities, nerve tortuosities, and age were analyzed with ANOVA and the Scheffe post hoc test. P-values less than 0.05 were considered to be statistically significant. The sample size was calculated based on published reports of subbasal nerve analyses for other ethnicities, and at least 76 eyes were requisite for a valid analysis. ${ }^{25-27}$

\section{Results}

Eighty eyes of 80 healthy subjects from Siriraj HealthScreening Center were enrolled. Their demographics showed a normal distribution; there were 20 participants in each age group, with an equal number of males and females (Table 1). The corneal nerve parameters (number and density of main nerve trunks, nerve branches, and total nerves) and the tortuosity analyzed by the two observers were compared. The validity of their data was checked via the ICC: it was found to be excellent (ICC =0.83). The parameters were then averaged and recorded. The overall number of the main nerve trunks, nerve branches, and total nerves were $4.47 \pm 0.13 /$ frame $\left(27.93 \pm 0.81 / \mathrm{mm}^{2}\right), 16.57$ $\pm 0.38 /$ frame $\left(103.56 \pm 2.37 / \mathrm{mm}^{2}\right)$, and $21.04 \pm 0.82 /$ frame $\left(131.50 \pm 5.12 / \mathrm{mm}^{2}\right)$, respectively. Meanwhile, the density of the main nerve trunks, nerve branches, and total nerves were $11.22 \pm 0.30 \mathrm{~mm} / \mathrm{mm}^{2}, 9.15 \pm 0.30 \mathrm{~mm} / \mathrm{mm}^{2}$, and $20.37 \pm 0.39 \mathrm{~mm} / \mathrm{mm}^{2}$, respectively. There was no statistically significant difference between the numbers and densities of the subbasal nerves of the population aged 20-60 years (all $\mathrm{p}>0.05$; Table 2). Regarding the tortuosity of the corneal nerves, the overall nerve tortuosity was grade 2.54 . The majority of the subjects aged 20-40 years showed a low-grade tortuosity ( $\leq$ grade 2 ). In contrast, nerve tortuosity increased among subjects aged over 40 years (Table 3 and Figure 1). However, there was no statistically significant difference in the nerve tortuosity of all subjects. No correlations between subbasal nerve density, tortuosity, and age (range: 20-60 years) were found $(\mathrm{p}>0.05)$.

\section{Discussion}

This study used laser in vivo confocal microscopy to analyze the corneal subbasal nerve characteristics of a healthy population aged $20-60$ years. The results revealed no significant differences in the subbasal nerve densities and tortuosity of the subjects in this age range. However, most of the individuals aged over 40 years demonstrated a higher degree of tortuosity of their corneal nerves than the younger ones. No significant correlations between subbasal nerve density, nerve tortuosity, and age were established.

The advent of the in vivo analysis of corneal microstructures that has resulted from advances in the technology of confocal microscopy has produced a wealth of informative data on normal and pathological corneas. The subbasal nerve plexus is one of the most distinct structures demonstrated by corneal IVCM. As a result, it has been widely studied, yielding an immense comprehension of corneal innervation. The confocal analysis of the subbasal nerves has gained considerable interest from investigators given that the relationship between corneal innervation and several diseases is increasingly being revealed. ${ }^{26,28}$ As to corneal diseases, IVCM is the mainstay tool for detecting changes in the corneal nerves in patients with NK, thereby facilitating the early diagnosis of 
Table 2 Corneal Subbasal Nerve Parameters of 80 Healthy Subjects, Classified into 4 Age Groups

\begin{tabular}{|c|c|c|c|c|c|}
\hline & \multicolumn{4}{|l|}{ Age (Years) } & \multirow[t]{2}{*}{ Total } \\
\hline & $20-30$ & $>30-40$ & $>40-50$ & $>50-60$ & \\
\hline \multicolumn{6}{|l|}{ Number of nerves } \\
\hline Main trunks (No./frame) & $4.58 \pm 0.24$ & $4.04 \pm 0.27$ & $4.71 \pm 0.18$ & $4.53 \pm 0.31$ & $4.47 \pm 0.13$ \\
\hline Main trunks $\left(\mathrm{No} . / \mathrm{mm}^{2}\right)$ & $28.62 \pm 1.50$ & $25.25 \pm 1.68$ & $29.43 \pm 1.12$ & $28.31 \pm 1.93$ & $27.93 \pm 0.81$ \\
\hline Nerve branches (No./frame) & $14.75 \pm 0.47$ & $15.93 \pm 0.75$ & $19.36 \pm 0.80$ & $16.53 \pm 0.93$ & $16.57 \pm 0.38$ \\
\hline Nerve branches (No./mm²) & $92.18 \pm 2.93$ & $99.56 \pm 4.68$ & $121.00 \pm 1.33$ & $\mid 03.31 \pm 5.81$ & $103.56 \pm 2.37$ \\
\hline Total nerves (No./frame) & $19.33 \pm 1.09$ & $19.97 \pm 1.81$ & $24.07 \pm 1.94$ & $21.06 \pm 1.65$ & $21.04 \pm 0.82$ \\
\hline Total nerves (No./mm²) & $|20.8| \pm 6.8 \mid$ & $|24.8| \pm \mid 1.31$ & $150.43 \pm 12.12$ & $|3| .62 \pm 10.3 \mid$ & $13 \mid .5 \pm 5.12$ \\
\hline \multicolumn{6}{|l|}{ Density of nerves } \\
\hline Main nerves $\left(\mathrm{mm} / \mathrm{mm}^{2}\right)$ & $1 \mathrm{I} .35 \pm 0.6 \mathrm{I}$ & $10.19 \pm 0.69$ & $11.89 \pm 0.38$ & $\mathrm{II} .44 \pm 0.66$ & $11.22 \pm 0.30$ \\
\hline Nerve branches $\left(\mathrm{mm} / \mathrm{mm}^{2}\right)$ & $9.00 \pm 0.39$ & $8.95 \pm 0.60$ & $9.67 \pm 0.77$ & $9.00 \pm 0.66$ & $9.15 \pm 0.30$ \\
\hline Total nerves $\left(\mathrm{mm} / \mathrm{mm}^{2}\right)$ & $20.35 \pm 0.69$ & $19.14 \pm 0.90$ & $21.56 \pm 0.83$ & $20.44 \pm 0.64$ & $20.37 \pm 0.39$ \\
\hline
\end{tabular}

Notes: Values are presented as mean \pm standard error of mean (SEM). Independent $t$-test was performed to analyze the difference of subbasal nerve number and density between the age groups. Statistical significance when $p<0.05$ compared between the age groups; all $p>0.05$.

Table 3 Grading of Corneal Subbasal Nerve Tortuosity of 80 Healthy Subjects, Classified into 4 Age Groups

\begin{tabular}{|c|c|c|c|c|c|}
\hline \multirow[t]{2}{*}{ Grading of Nerve Tortuosity } & \multicolumn{4}{|c|}{ Age (Years) } & \multirow[t]{2}{*}{ Total } \\
\hline & $20-30$ & $>30-40$ & $>40-50$ & $>50-60$ & \\
\hline Grade 0 & - & - & - & - & - \\
\hline Grade I & 4 & 4 & I & 2 & 11 \\
\hline Grade 2 & 10 & 11 & 7 & 4 & 32 \\
\hline Grade 3 & 3 & 4 & 8 & 5 & 20 \\
\hline Grade 4 & 2 & 2 & 5 & 8 & 17 \\
\hline
\end{tabular}

Notes: The tortuosity of corneal subbasal nerves was graded in accordance with the classification proposed by Oliviera-Soto et al.

$\mathrm{NK}$, the determination of a prognosis, and the evaluation of the efficacy of treatment. ${ }^{29}$ Bilateral corneal nerve loss in patients with unilateral herpes zoster ophthalmicus and herpes simplex keratitis is also uncovered with IVCM, ${ }^{30-32}$ as are the cases with other forms of infectious keratitis. ${ }^{33,34}$ Moreover, altered corneal nerve parameters have been found in several conditions of ocular surface diseases, such as limbal stem cell deficiency, ${ }^{35}$ dry eye ${ }^{36,37}$ meibomian gland dysfunction, ${ }^{38}$ vernal keratoconjunctivitis, ${ }^{39}$ and recurrent corneal erosion. ${ }^{40}$ Beyond the eye, a link between corneal innervation and the central regulation of the nervous system has also been proposed, thereby offering a possible pathophysiological cause of the pain experienced by patients with corneal allodynia. ${ }^{41}$ As for systemic conditions, a number of published studies have shown the relevance of the pathological changes in systemic neuropathy-related diseases to corneal nerve alterations. ${ }^{42-46}$ It therefore appears likely that many more disclosures can be expected from future research.
Up to present, three main IVCM devices have been used in clinical practice: the tandem scanning confocal microscope (TSCM), the slit-scanning confocal microscope (SSCM), and the laser scanning confocal microscope (LSCM). ${ }^{13,47}$ Although the TSCM is no longer produced, the latter two remain commercially available and are more widely used. The properties of the SSCM differ entirely from those of the LSCM, resulting in dissimilar advantages and disadvantages. ${ }^{48}$ Generally, the SSCM is considered user-friendly as it can be conveniently operated in either an automatic or semi-automatic mode to quickly achieve $500 \times$ confocal images of scanned objects with $26 \mu \mathrm{m}$ axial resolution. ${ }^{49}$ The device uses a halogen lamp as the light source, which provides a broad beam and adjustable brightness during corneal scanning. With this slightly wide field of illumination, the SSCM induces the magnitude of the backscattering of the projected light when it hits the intracorneal structures, especially the opaque areas. Because the image resolution is 


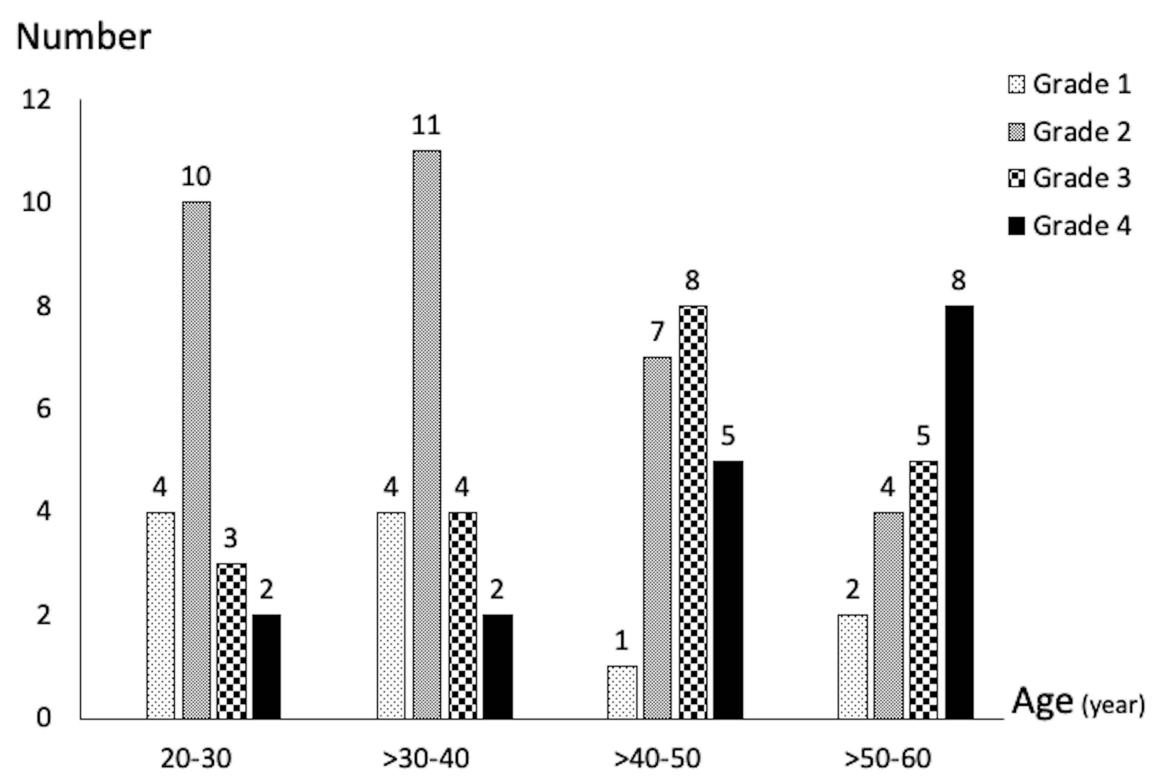

Figure I The bar graph demonstrates the patterns of corneal subbasal nerve tortuosity in different age groups.

somewhat degraded by the adjacent light scattering, the confocal images derived from an SSCM may lose their contrast and sharpness. As to the LSCM, although it can be operated manually or semiautomatically, more complicated techniques need to be used than with the SSCM. The LSCM employs the tiny beam of a $670 \mathrm{~nm}$ laser as its light source; while this raises a concern about the theoretical possibility of epithelial injury, no instances have yet been reported. ${ }^{2}$ Given that the high-intensity light beam illuminates a relatively small scanning area, the confocal images of the cornea provided by the LSCM have a higher resolution with lower interference from the surrounding backscattered light compared to images obtained from the SSCM. The latest version of the LSCM (Heidelberg Retina Tomograph 3 with Rostock Cornea Module [HRT3/RCM], Heidelberg Engineering GmbH., Heidelberg, Germany) can produce high-contrast, $800 \times$ images of scanned objects with a 5-7 $\mu \mathrm{m}$ axial resolution.${ }^{2,49}$ Although both the SSCM and LSCM share the same principles of confocal-imaging devices, the dissimilarities between the two IVCM systems contribute to evident differences in the quality of their confocal images (Figure 2). Accordingly, the quantified analyses of intracorneal cells and/or structures obtained from different confocal technologies are neither comparable nor interchangeable.

In vivo confocal analysis of the corneal subbasal nerves essentially refers to the study of nerve density and morphology. In clinical practice, acquiring normative data of corneal nerve characteristics via a standard IVCM analysis is a prerequisite for establishing a baseline to be later compared with the nerve findings detected in various diseases. Previous studies have shown varying densities of normal subbasal nerve plexus using different types of IVCM conducted in diverse ethnic populations. ${ }^{26,50}$ Only the densities derived from research employing LSCMs were close to our results, and the densities were higher than those obtained by the studies using SSCMs and TSCMs. This is not surprising as LSCMs provide a better image contrast and resolution than the other two systems, thereby allowing nerve fibers to be more easily visualized and quantified than with the alternative IVCM devices.

Nowadays, the IVCM nerve analysis has been popularly performed using both fully automated (such as ACCMetrics $^{51}$ and deepNerve metrics ${ }^{52}$ ) and semiautomated technique (such as NeuronJ, which we used in the current study). Although the fully automated software seems to be more advantageous in terms of speed, objectivity, and consistency over the semiautomated ones. Fully automated metrics are not acquired and implemented for a real practice in most of the centers. Moreover, there were several IVCM studies affirmed that there was no difference of subbasal nerve analysis between manual, semiautomated and fully automated methods. ${ }^{19,51,52}$ Therefore, we facilitated the nerve analysis for general application by owing the more available semiautomated program. Furthermore, we tried to minimize the inter-rater variability of the analysis by performing ICC of the two raters 

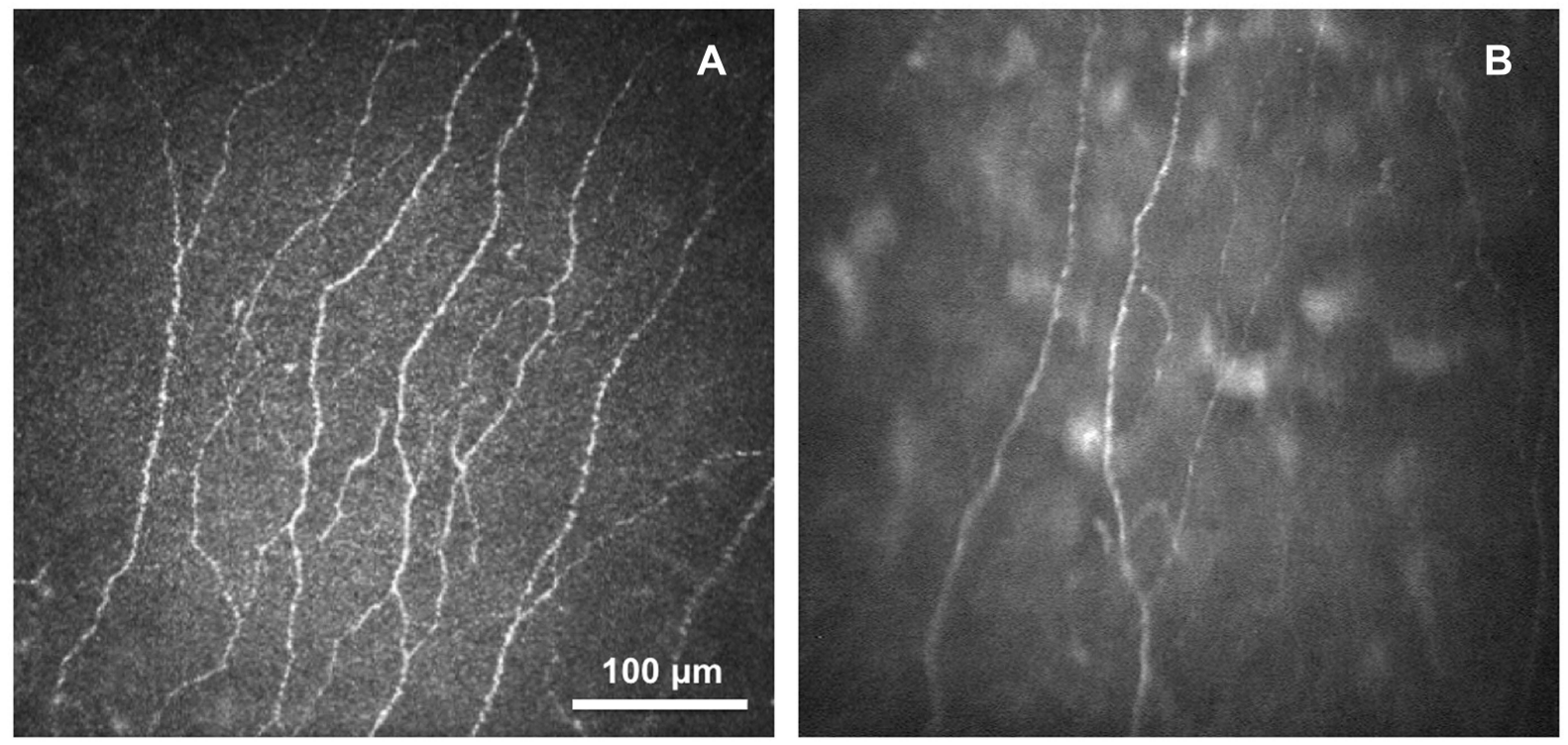

Figure 2 Confocal images of corneal subbasal nerves obtained from 2 types of confocal microscope: (A) Laser scanning confocal microscope (LSCM) and (B) Slit-scanning confocal microscope (SSCM).

before performing the actual data analysis. We set the threshold of ICC at 0.75 as the higher value than this was considered an excellent agreement between raters. ${ }^{22}$ Fortunately, our two raters achieved the ICC value of 0.83 .

In the current study, we did not find any significant differences in the subbasal nerve densities of the four age groups, and consistent with some earlier reports, no correlation between corneal nerve density and age was observed. ${ }^{17,18,42,53,54}$ However, some investigators have revealed contradictory results as they identified that significant nerve loss was related to increasing age. ${ }^{19-21,23,55,56}$ These studies included subjects older than the age of 60 , which was beyond the range of our study group. The rationale that we recruited subjects under 60 years old was to analyze the normal nerve characteristics and to preclude the results of nerve analysis affected from degenerative changes in the elderly. Since Parissi et $\mathrm{al}^{19}$ and Dehghani et $\mathrm{al}^{18}$ have reported a gradual nerve decline with age in their series.

The discordance between the results of the studies might also reflect the differences in the following areas: the IVCM systems utilized; the nerve quantification methods employed; the exact location of the subbasal nerve analysis; and the age ranges of the subjects, which were entirely inconsistent across the studies. In the case of the present study, we took several actions to standardize the data. At the outset, all included subjects were proven to be healthy by undergoing blood screening tests and completing an eye examination to rule out any concealed causes of nerve deterioration. In addition, the sample size was made sufficiently large for analysis purposes, and the number of subjects was equal in each age group. Moreover, the distribution of the subjects' ages in each subgroup was verified with a normality test. This allowed us to develop a good perspective of the nerve characteristics of both the overall study cohort as well as the specific age groups. Furthermore, an LSCM was chosen to ensure that there would be favorable visualization of the subbasal nerves and accurate nerve quantification. Lastly, before reporting the outcomes, the reliability of the results obtained from the two experienced observers was verified; with an ICC value of 0.83 , it was deemed to be excellent.

Regarding the evaluation of nerve tortuosity, we followed a manual classification system described by Oliveira-Soto et al. ${ }^{23}$ In fact, several publications have proposed distinct methodologies for the assessment of the tortuosity of subbasal nerves. ${ }^{57-}$ ${ }^{59}$ Unfortunately, most of those methods need sophisticated software and can only be performed in some specific centers. Therefore, we pursued a practical method that can be conveniently applied in general practice. Our results revealed that the overall nerve tortuosity of a healthy population was grade 2.54 , which was higher than the results of other reports. ${ }^{26,60,61}$ This can be explained by the variances in the techniques for nerve morphology evaluation and in the different confocal-scanning areas of the studies, which could not be definitely identified. Misra et al demonstrated a high tortuosity of subbasal nerves 
configurated in a whorl pattern and located slightly inferocentrally of the cornea. ${ }^{46}$ However, this did not affect the density of the subbasal nerves as the amount of nerves in the central cornea has been demonstrated by another study to be equal to that in the midcentral region. ${ }^{62}$ Furthermore, we noticed a trend towards a rise in nerve tortuosity in the two oldest age groups relative to the younger groups, but there was no statistically significant correlation between nerve tortuosity and age. It is presumed that there might be some degree of degenerative change influencing this alteration but not enough to affect subbasal nerve density in people aged over 40 years. Some limitations of this study were 1) we assumed that the findings from the central cornea could be extrapolated to represent the whole of the corneal subbasal nerves. The results would, of course, be more accurate if the subbasal nerves throughout the entire cornea were analyzed; 2) in the present study, only Thai ethnicity was included. The invariable ethnicity allowed in the study should be taken into consideration when the results would be applied in clinical practice. However, a previous study demonstrated that there was no significant difference of subbasal nerve density between ethnic groups when employing a similar confocal technique. ${ }^{11}$ and 3) the present study did not include subjects older than the age of 60 as being aware of the effect of degenerative change. Therefore, our results were not exactly in unison with other studies due to the difference of participants' age range.

\section{Conclusion}

This study analyzes the characteristics of corneal subbasal nerve of an adult healthy population, using laser in vivo confocal microscopy. We did not find any significant differences in the subbasal nerve densities and tortuosities of the subjects, who were aged 20-60 years. However, the tortuosity of the corneal nerves seems to increase in people aged over 40 years. A definite correlation between subbasal nerve density, nerve tortuosity, and age was not determined. With its carefully considered study design, the results of the corneal subbasal nerve analysis in the present research can be justifiably used as primary references and compared with corneal nerve findings in patients with diseases related to corneal nerve alterations.

\section{Funding}

This study is supported by the Research Development Fund of the Faculty of Medicine Siriraj Hospital, Mahidol University (No. R016031027). The funding organization has no role in the design or conduct of this study.

\section{Disclosure}

The authors report no conflicts of interest and have no proprietary interest in any of the materials mentioned in this article.

\section{References}

1. Bonini S, Rama P, Olzi D, et al. Neurotrophic keratitis. Eye (Lond). 2003;17(8):989-995. doi:10.1038/sj.eye.6700616

2. Cruzat A, Pavan-Langston D, Hamrah P. In vivo confocal microscopy of corneal nerves: analysis and clinical correlation. Semin Ophthalmol. 2010;25(5-6):171-177.

3. Dua HS, Said DG, Messmer EM, et al. Neurotrophic keratopathy. Prog Retin Eye Res. 2018;66:107-131.

4. Sacchetti M, Lambiase A. Diagnosis and management of neurotrophic keratitis. Clin Ophthalmol. 2014;8:571-579.

5. Liu Q, Xu Z, Xu Y, et al. Changes in corneal dendritic cell and subbasal nerve in long-term contact lens wearers with dry eye. Eye Contact Lens. 2020;46(4):238-244.

6. Cruzat A, Qazi Y, Hamrah P. In vivo confocal microscopy of corneal nerves in health and disease. Ocul Surf. 2017;15(1):15-47.

7. Lockwood A, Hope-Ross M, Chell P. Neurotrophic keratopathy and diabetes mellitus. Eye (Lond). 2006;20(7):837-839.

8. Lum E, Murphy PJ. Effects of ambient humidity on the CochetBonnet aesthesiometer. Eye (Lond). 2018;32(10):1644-1651.

9. Ornek N, Dag E, Ornek K. Corneal sensitivity and tear function in neurodegenerative diseases. Curr Eye Res. 2015;40(4):423-428.

10. Hamrah P, Cruzat A, Dastjerdi MH, et al. Corneal sensation and subbasal nerve alterations in patients with herpes simplex keratitis: an in vivo confocal microscopy study. Ophthalmology. 2010;117 (10):1930-1936. doi:10.1016/j.ophtha.2010.07.010

11. Patel DV, McGhee CN. Contemporary in vivo confocal microscopy of the living human cornea using white light and laser scanning techniques: a major review. Clin Exp Ophthalmol. 2007;35(1):71-88.

12. Villani E, Baudouin C, Efron N, et al. In vivo confocal microscopy of the ocular surface: from bench to bedside. Curr Eye Res. 2014;39 (3):213-231.

13. Stachs O, Guthoff RF, Aumann S. In vivo confocal scanning laser microscopy. In: Bille JF, editor. High Resolution Imaging in Microscopy and Ophthalmology: New Frontiers in Biomedical Optics. Switzerland: Springer; 2019:263-284.

14. Chirapapaisan C, Abbouda A, Jamali A, et al. In vivo confocal microscopy demonstrates increased immune cell densities in corneal graft rejection correlating with signs and symptoms. Am $J$ Ophthalmol. 2019;203:26-36.

15. Prabhasawat P, Luangaram A, Ekpo P, et al. Epithelial analysis of simple limbal epithelial transplantation in limbal stem cell deficiency by in vivo confocal microscopy and impression cytology. Cell Tissue Bank. 2019;20(1):95-108.

16. Chidambaram JD, Prajna NV, Palepu S, et al. In vivo confocal microscopy cellular features of host and organism in bacterial, fungal, and acanthamoeba keratitis. Am J Ophthalmol. 2018;190:24-33.

17. Erie JC, McLaren JW, Hodge DO, et al. The effect of age on the corneal subbasal nerve plexus. Cornea. 2005;24(6):705-709.

18. Dehghani C, Pritchard N, Edwards K, et al. Morphometric stability of the corneal subbasal nerve plexus in healthy individuals: a 3-year longitudinal study using corneal confocal microscopy. Invest Ophthalmol Vis Sci. 2014;55(5):3195-3199.

19. Parissi M, Karanis G, Randjelovic S, et al. Standardized baseline human corneal subbasal nerve density for clinical investigations with laser-scanning in vivo confocal microscopy. Invest Ophthalmol Vis Sci. 2013;54(10):7091-7102.

20. Roszkowska AM, Wylegala A, Gargano R, et al. Impact of corneal parameters, refractive error and age on density and morphology of the subbasal nerve plexus fibers in healthy adults. Sci Rep. 2021;11 (1):6076. 
21. Tavakoli M, Ferdousi M, Petropoulos IN, et al. Normative values for corneal nerve morphology assessed using corneal confocal microscopy: a multinational normative data set. Diabetes Care. 2015;38 (5):838-843.

22. Meijering E, Jacob M, Sarria JC, et al. Design and validation of a tool for neurite tracing and analysis in fluorescence microscopy images. Cytometry A. 2004;58(2):167-176.

23. Oliveira-Soto L, Efron N. Morphology of corneal nerves using confocal microscopy. Cornea. 2001;20(4):374-384.

24. Rosner B. The Intraclass Correlation Coefficient. In: Rosner B, editor. Fundamentals of Biostatistics. 5th ed. USA: Pacific Grove; 2000:562-563.

25. Bonett DG. Sample size requirements for estimating intraclass correlations with desired precision. Stat Med. 2002;21(9):1331-1335.

26. Patel DV, McGhee CN. In vivo confocal microscopy of human corneal nerves in health, in ocular and systemic disease, and following corneal surgery: a review. Br J Ophthalmol. 2009;93(7):853-860.

27. Dieckmann G, Pupe C, Nascimento OJ. Corneal confocal microscopy in a healthy Brazilian sample. Arq Neuropsiquiatr. 2016;74(1):10-17.

28. Al-Aqaba MA, Dhillon VK, Mohammed I, et al. Corneal nerves in health and disease. Prog Retin Eye Res. 2019;73:100762.

29. Fung SSM, Catapano J, Elbaz U, et al. In vivo confocal microscopy reveals corneal reinnervation after treatment of neurotrophic keratopathy with corneal neurotization. Cornea. 2018;37(1):109-112.

30. Hamrah P, Cruzat A, Dastjerdi MH, et al. Unilateral herpes zoster ophthalmicus results in bilateral corneal nerve alteration: an in vivo confocal microscopy study. Ophthalmology. 2013;120(1):40-47.

31. Cavalcanti BM, Cruzat A, Sahin A, et al. In vivo confocal microscopy detects bilateral changes of corneal immune cells and nerves in unilateral herpes zoster ophthalmicus. Ocul Surf. 2018;16(1):101111.

32. Moein HR, Kheirkhah A, Muller RT, et al. Corneal nerve regeneration after herpes simplex keratitis: a longitudinal in vivo confocal microscopy study. Ocul Surf. 2018;16(2):218-225.

33. Cruzat A, Schrems WA, Schrems-Hoesl LM, et al. Contralateral clinically unaffected eyes of patients with unilateral infectious keratitis demonstrate a sympathetic immune response. Invest Ophthalmol Vis Sci. 2015;56(11):6612-6620.

34. Muller RT, Abedi F, Cruzat A, et al. Degeneration and regeneration of subbasal corneal nerves after infectious keratitis: a longitudinal in vivo confocal microscopy study. Ophthalmology. 2015;122 (11):2200-2209.

35. Bhattacharya P, Edwards K, Harkin D, et al. Central corneal basal cell density and nerve parameters in ocular surface disease and limbal stem cell deficiency: a review and meta-analysis. $\mathrm{Br} J$ Ophthalmol. 2020;104(12):1633-1639.

36. Matsumoto Y, Ibrahim OMA. Application of in vivo confocal microscopy in dry eye disease. Invest Ophthalmol Vis Sci. 2018;59(14): DES41-DES7.

37. Labbe A, Liang Q, Wang Z, et al. Corneal nerve structure and function in patients with non-sjogren dry eye: clinical correlations. Invest Ophthalmol Vis Sci. 2013;54(8):5144-5150.

38. Azizi S, Ucak T, Yasar I, et al. Evaluation of the corneal layers in meibomian-gland-dysfunction-related dry eye by in vivo slit-scanning confocal microscopy. Semin Ophthalmol. 2017;32(3):377-383.

39. Leonardi A, Lazzarini D, Bortolotti M, et al. Corneal confocal microscopy in patients with vernal keratoconjunctivitis. Ophthalmology. 2012;119(3):509-515.

40. Trufanov SV, Tekeeva LY, Surnina ZV, et al. Morphological changes in the cornea of patients with recurrent corneal erosion after diamond burr polishing of Bowman's membrane. Vestn Oftalmol. 2019;135 (5):24-30.

41. Hamrah P, Qazi Y, Shahatit B, et al. Corneal nerve and epithelial cell alterations in corneal allodynia: an in vivo confocal microscopy case series. Ocul Surf. 2017;15(1):139-151.
42. Wu T, Ahmed A, Bril V, et al. Variables associated with corneal confocal microscopy parameters in healthy volunteers: implications for diabetic neuropathy screening. Diabet Med. 2012;29(9):e297303.

43. Oudejans L, He X, Niesters M, et al. Cornea nerve fiber quantification and construction of phenotypes in patients with fibromyalgia. Sci Rep. 2016;6:23573.

44. De Clerck EEB, Schouten J, Berendschot T, et al. Reduced corneal nerve fibre length in prediabetes and type 2 diabetes: the Maastricht Study. Acta Ophthalmol. 2020;98(5):485-491.

45. Kamran S, Khan A, Salam A, et al. Cornea: a window to white matter changes in stroke; corneal confocal microscopy a surrogate marker for the presence and severity of white matter hyperintensities in ischemic stroke. J Stroke Cerebrovasc Dis. 2020;29(3):104543.

46. Misra SL, Kersten HM, Roxburgh RH, et al. Corneal nerve microstructure in Parkinson's disease. J Clin Neurosci. 2017;39:53-58.

47. Petroll WM, Robertson DM. In vivo confocal microscopy of the cornea: new developments in image acquisition, reconstruction, and analysis using the HRT-Rostock corneal module. Ocul Surf. 2015;13 (3):187-203.

48. Stanca TH, Tabacaru B, Celea C. Correlations between confocal microscopy and histological aspects of normal cornea. Rom J Ophthalmol. 2015;59(1):19-23.

49. Tavakoli M, Hossain P, Malik RA. Clinical applications of corneal confocal microscopy. Clin Ophthalmol. 2008;2(2):435-445.

50. Niederer RL, McGhee CN. Clinical in vivo confocal microscopy of the human cornea in health and disease. Prog Retin Eye Res. 2010;29 (1):30-58.

51. Dehghani C, Pritchard N, Edwards K, et al. Fully automated, semiautomated, and manual morphometric analysis of corneal subbasal nerve plexus in individuals with and without diabetes. Cornea. 2014;33(7):696-702.

52. Oakley JD, Russakoff DB, McCarron ME, et al. Deep learning-based analysis of macaque corneal sub-basal nerve fibers in confocal microscopy images. Eye Vis (Lond). 2020;7:27.

53. Grupcheva CN, Wong T, Riley AF, et al. Assessing the sub-basal nerve plexus of the living healthy human cornea by in vivo confocal microscopy. Clin Exp Ophthalmol. 2002;30(3):187-190.

54. Patel DV, Tavakoli M, Craig JP, et al. Corneal sensitivity and slit scanning in vivo confocal microscopy of the subbasal nerve plexus of the normal central and peripheral human cornea. Cornea. 2009;28 (7):735-740.

55. Niederer RL, Perumal D, Sherwin T, et al. Age-related differences in the normal human cornea: a laser scanning in vivo confocal microscopy study. Br J Ophthalmol. 2007;91(9):1165-1169.

56. Marco B, Alessandro R, Philippe F, et al. The effect of aging on nerve morphology and substance $P$ expression in mouse and human corneas. Invest Ophthalmol Vis Sci. 2018;59(13):5329-5335.

57. Annunziata R, Kheirkhah A, Aggarwal S, et al. Two-dimensional plane for multi-scale quantification of corneal subbasal nerve tortuosity. Invest Ophthalmol Vis Sci. 2016;57(3):1132-1139.

58. Kallinikos P, Berhanu M, O'Donnell C, et al. Corneal nerve tortuosity in diabetic patients with neuropathy. Invest Ophthalmol Vis Sci. 2004;45(2):418-422.

59. Lum E, Golebiowski B, Swarbrick HA. Changes in corneal subbasal nerve morphology and sensitivity during orthokeratology: recovery of change. Ocul Surf. 2017;15(2):236-241.

60. Mocan MC, Durukan I, Irkec M, et al. Morphologic alterations of both the stromal and subbasal nerves in the corneas of patients with diabetes. Cornea. 2006;25(7):769-773.

61. Zhang M, Chen J, Luo L, et al. Altered corneal nerves in aqueous tear deficiency viewed by in vivo confocal microscopy. Cornea. 2005;24 (7):818-824.

62. Muller LJ, Vrensen GF, Pels L, et al. Architecture of human corneal nerves. Invest Ophthalmol Vis Sci. 1997;38(5):985-994. 


\section{Publish your work in this journal}

Clinical Ophthalmology is an international, peer-reviewed journal covering all subspecialties within ophthalmology. Key topics include: Optometry; Visual science; Pharmacology and drug therapy in eye diseases; Basic Sciences; Primary and Secondary eye care; Patient Safety and Quality of Care Improvements. This journal is indexed on PubMed
Central and CAS, and is the official journal of The Society of Clinical Ophthalmology (SCO). The manuscript management system is completely online and includes a very quick and fair peer-review system, which is all easy to use. Visit http://www.dovepress.com/ testimonials.php to read real quotes from published authors.

Submit your manuscript here: https://www.dovepress.com/clinical-ophthalmology-journal 